\title{
MODELING FAINT GALAXY COUNTS
}

\author{
B. Guiderdoni ${ }^{1}$ and B. Rocca-Volmerange ${ }^{1,2}$ \\ ${ }^{1}$ Institut d'Astrophysique de Paris $\quad{ }^{2}$ Laboratoire René Bernas \\ 98 bis Boulevard Arago \\ F-75014 Paris \\ Bât. 108 \\ Université Paris XI \\ France \\ F-91405, Orsay \\ France
}

In this paper, we summarize the results of an analysis of faint galaxy counts presented in Guiderdoni and Rocca-Volmerange (1989). We choose to restrict our analysis here to the examination of pure luminosity evolution.

The intrinsic evolution can be analyzed by means of a model of spectrophotometric evolution. Such a model allows us to compute evolving synthetic spectra of galaxies from a minimum set of assumptions about star formation history and apparent magnitudes and colors that take into account the entangling of the cosmological and evolutionary effects in a consistent way. In this paper, we use our model of spectrophotometric evolution (Guiderdoni and Rocca-Volmerange 1987; Rocca-Volmerange and Guiderdoni 1988).

Figure 1 shows the influence of varying $z_{\text {for }}$ for $q_{0}=0.05$. Large values of $z_{\text {for }}$ are required to fit the faint galaxy counts. The value $z_{\text {for }}=5$ predicts a bump around $B_{J} \equiv J^{+}=24$ (not shown in the data). The value $z_{\text {for }}=2$ does not reproduce the slope fainter than $\mathrm{J}^{+}=20$, and the predicted faint-magnitude plateau is strongly discrepant with the data. Figure 2 shows the predictions with various $q_{0}$ and $z_{\text {for }}=30$. The various predictions without evolution are shown the predictions with evolution are much more sensitive to $q_{0}$ than are the predictions without evolution. The plateau occurs at lower levels for higher $q_{0}$, with a factor of $\sim 4$ shift in the counts between $q_{0}=0$ and $q_{0}=0.5$. Small values of $q_{0}$ are strictly required to fit the counts. The value $q_{0}=0.05$ fits well from the bright end down to $J^{+} \approx 27$.

We use our model to compute the extragalactic background light (EBL) produced by galaxies. Figure 3 shows the predictions for various values of $z_{\text {for }}$ and $q_{0}$ taking into account that evolution increases the EBL by a factor of $\sim 2$. With these standard scenarios of galactic evolution, the predicted EBL is clearly well below the observational limits in the visible and the near-IR.

\section{REFERENCES}

Dube, R. R., Wickes, W. C., and Wilkinson, D. T. 1979, Ap. J., 232, 333.

Guiderdoni, B. and Rocca-Volmerange, B. 1987, Astron. Astrophys., 186, 1.

Guiderdoni, B. and Rocca-Volmerange, B. 1989, Astron. Astrophys., in press.

Matsumoto, T. Akiba, M. and Murakami, H. 1988, Ap. J., 332, 575.

Paresce, F. and Jakobsen, P. 1980, Nature, 288, 119.

Rocca-Volmerange, B. and Guiderdoni, B. 1988, Astron. Astrophys. Suppl. Series, 75, 93.

Toller, G. N. 1983, Ap. J. Letters, 266, L79. 


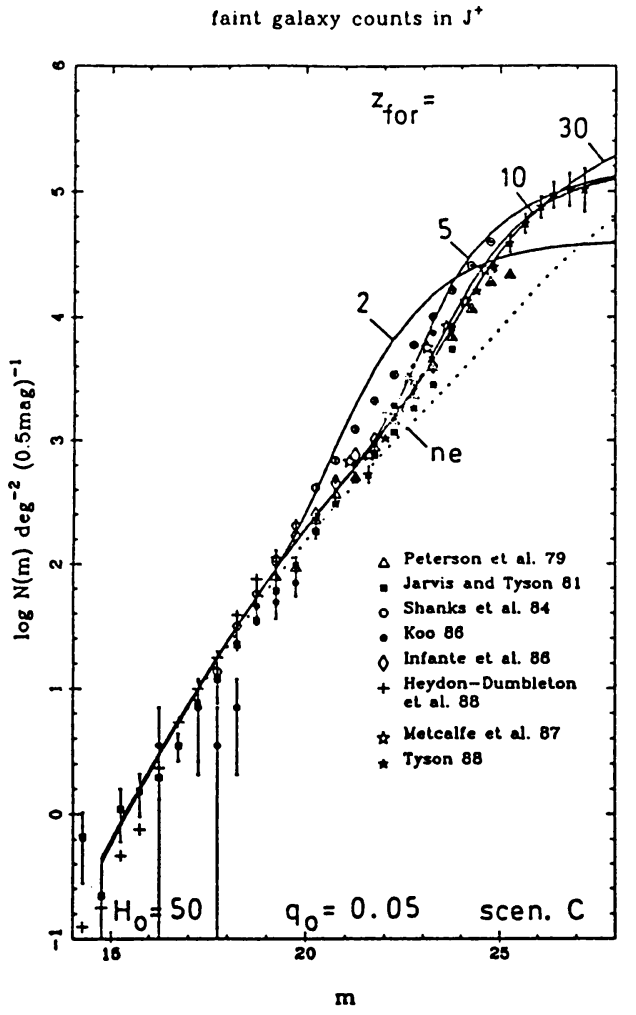

Figure 1. Influence of $z_{\text {for }}$ on the faint galaxy counts, $q_{0}=0.05$. Solid lines: with evolution. Dotted line: without evolution. Large values of $z_{\text {for }}$ are required to fit the slope and the plateau when fainter than $B_{J} \equiv J^{+} \approx 25$.

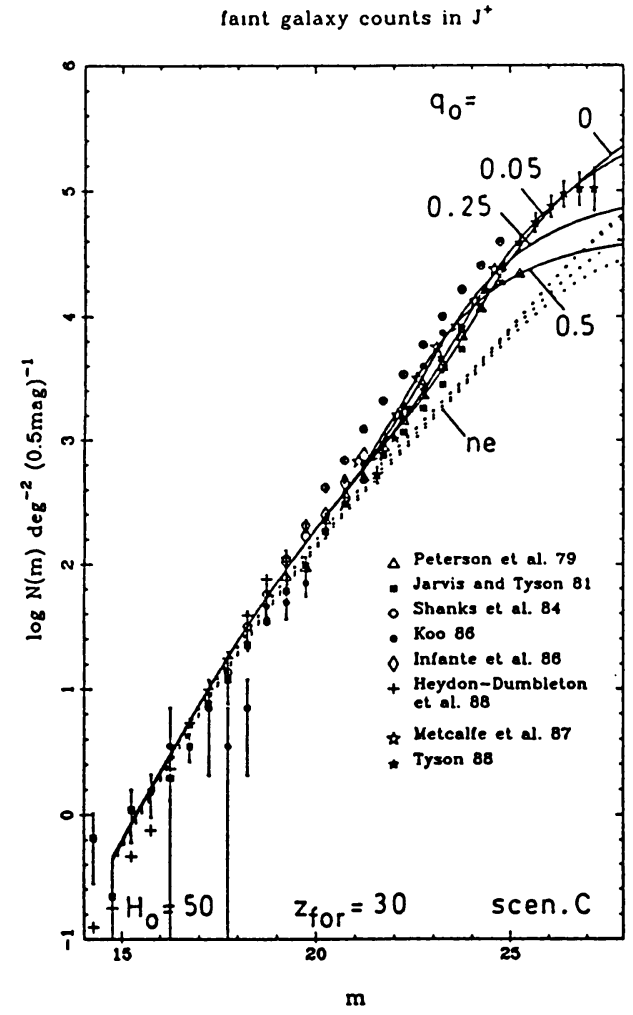

Figure 2. Influence of $q_{0}$ on the faint galaxy counts, $z_{\text {for }}=30$. Solid lines: with evolution. Dotted lines: without evolution. Small values of $q_{0}$ are required to fit the slope and the plateau when fainter than $B_{J} \equiv J^{+} \approx 25$.

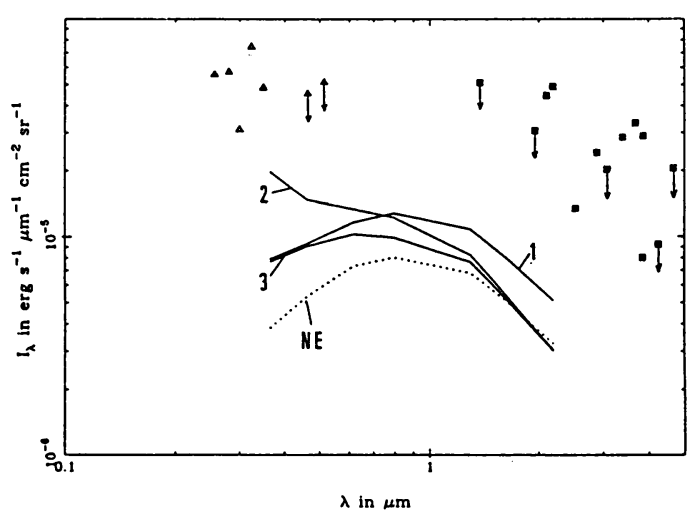

Figure 3. Prediction of the EBL. Solid lines: with evolution. Dotted line: without evolution. (1) $q_{0}=0.05$ and $z_{\text {for }}=30$. (2) $q_{0}=$ 0.05 and $z_{\text {for }}=2$. (3) $q_{0}=0.5$ and $z_{\text {for }}=30$. Observations: near-IR (black squares), Matsumoto, Akiba, and Murakami (1988); visible (blade triangles), Dube et al. (1977), Toller (1983); near-UV (open triangles), compilation by Paresce and Jakobsen (1980). 